\title{
ANÁLISIS DEL COMPORTAMIENTO DE SUELOS DE ALTA PLASTICIDAD CON LA ADICIÓN DEL MATERIAL DE RESIDUO EN LA FABRICACIÓN DE LADRILLO CERÁMICO
}

\section{ANALYSIS OF THE BEHAVIOR OF HIGH PLASTICITY FLOORS WITH THE ADDITION OF RESIDUE MATERIAL IN THE MANUFACTURE OF CERAMIC BRICK}

\author{
MSc. Romel Jesús Gallardo Amaya, Dr. Oscar Andrés Cuanalo Campos, \\ Ing. Leidy Johana Quintero Lemus , Est. Angie Alejandra Muñoz, \\ Est. Ciro Andrey Martinez
}

\author{
Universidad Francisco de Paula Santander Ocaña, Facultad de Ingenierías, Grupo de \\ Investigación en Geotecnia y Medio Ambiente, GIGMA \\ Vía Acolsure, Sede el Algodonal, Ocaña, Norte de Santander, Colombia. \\ 5690088 ext. 509. \\ \{rjgallardoa, oscarcuanalo, ljquinterole, amunos, amartinez\}@ufpso.edu.co
}

\begin{abstract}
Resumen: En este proyecto de investigación se usó el residuo del ladrillo proveniente de la Ladrillera Ocaña, como un material alternativo para la adecuación de la subrasante de vías terciarias, la cual en muchas ocasiones está constituida por materiales de alta plasticidad que en épocas de invierno generan problemas de transitabilidad; siendo estas vías de gran importancia ya que permiten la comunicación a zonas de producción agrícola. La metodología utilizada se desarrolló en un marco Descriptivo-Experimental, donde se evidencio que el uso del residuo de ladrillo como material alternativo arroja buen comportamiento para disminuir la plasticidad y aumentar el índice CBR del suelo.
\end{abstract}

Palabras clave: Alta plasticidad, material alternativo y Mejoramiento de suelos

\begin{abstract}
In this research project residual brick, from the Ladrillera Ocaña, was used as an alternative material for the adequacy of subgrade of tertiary roads, twhich in many occasions are constituted by high plasticity materials that in the rainy season generate transit problems; being these roads of great importance, because they allow the communication to high agricultural productivity. The applied methodology was developed in a Descriptive- Experimental frame, where the good behavior of the residual brick as an alternative to decrease plasticity and increase increase the soil CBR index.
\end{abstract}

Keywords: High plasticity, alternative material, Soil improvement.

\section{INTRODUCCIÓN}

"El suelo es el agregado no cementado de granos minerales y materia orgánica descompuesto junto con el líquido y gas que ocupan los espacios vacíos entre las partículas sólidas" (Das, 1999), éste se encarga de sostener las cargas transmitidas por la superficie de rodadura en las vías y debe encontrarse en condiciones tales que sea competente para resistirlas, cuando los suelos no poseen las propiedades adecuadas por sí solos, se opta por cambiar el suelo por otro que sí posea las propiedades adecuadas o por realizar una estabilización para modificar sus propiedades naturales (Carvajal et al, 2015). Una característica de los tiempos actuales, en lo que se refiere al mejoramiento del suelo de subrasante, es el desarrollo de nuevas técnicas y tratamientos tendientes a lograr el máximo aprovechamiento de propiedades y características; buena parte de estos esfuerzos se ha orientado hacia la producción de inducidores y modificadores de comportamiento de suelos y materiales. (Tamayo, 1985) 
Los estudios sobre estabilización y/o mejoramiento de suelos viene en aumento desde el año 2000, donde se han utilizado diferentes residuos de las industrias para mejorar las propiedades físicas, químicas y mecánicas de los suelos, logrando reutilizar estos materiales; los suelos estabilizados constituyen capas uniformes, de calidad, con mayor durabilidad e insensibles al agua (Murthy, 2002).

Según Caravajal (et al 2015), el método de mayor auge para la estabilización y mejoramiento de suelos ha sido el uso de cenizas volantes, superando en cantidad a otros métodos de estabilización y/o mejoramiento como los son el cemento, las escorias, el metacaolín, el humo de sílice, las cenizas de cascara de arroz, entre otros.

"Países como España han desarrollado normas para la estabilización de suelos a base de cemento y cal, al igual que la National Lime Association en Estados Unidos para la estabilización de suelos tratados con cal, en Europa el empleo de las cenizas de carbón se ha visto influenciada por nuevas normas y legislaciones medioambientales y en Colombia existe la normativa INVIAS, la cual establece requisitos para estabilizar suelos con emulsión asfáltica, cemento y concreto hidráulico" (Carvajal et al 2015).

El Instituto de Desarrollo Urbano (2005) de Colombia, publicó un manual para el diseño y construcción de capas estructurales de pavimentos estabilizadas mediante procesos químicos; La normativa para la construcción de vías en Colombia está establecida por el Instituto Nacional de Vías (INVIAS), la cual establece requisitos para la estabilización de vías, construcción y normas de ensayos para materiales de carreteras, pero no establece requisitos para la estabilización usando materiales alternativos de los residuos de industrias, los cuales podrían ser reutilizados como materiales estabilizantes.

El uso de los materiales alternativos puede convertirse en una nueva solución de estabilización y/o mejoramiento de suelos de subrasante para vías terciarias, debido a que estas cuentan con muy pocos recursos para su construcción y por esta razón se encuentran muy deterioradas debido al uso de agregados sin tratar o de la sub rasante como superficie de rodadura (MINTRANSPORTE, 2011).

Según el Ministerio de Transporte en el año 2011, Colombia contaba con $128.000 \mathrm{~km}$ de vías, de los cuales $111.364 \mathrm{~km}$ pertenecían a la red secundaria y terciaria, estos estaban distribuidos en un $73 \%$ a cargo de las entidades territoriales, $18 \%$ a cargo de la Nación a través de la Subdirección de la Red Terciaria y Férrea del INVIAS, y el $8.3 \%$ es del sector privado, para el año 2013 de acuerdo a las estadísticas reportadas por FEDESARROLLO (2013), la red vial de Colombia ya era de aproximadamente 165.403 kilómetros y la red terciaria representaba la mayor cantidad de kilómetros de la red nacional con 126.154 km, lo que ha mostrado el importante aumento de la construcción de vías en el país, teniendo un aumento del $48 \%$ entre el año 2011 y el año 2013 en total de vías. La red vial se encuentra en un estado crítico, sobre todo la red terciaria, estas se han venido deteriorando por la falta de mantenimiento debido a que los departamentos y la Nación cuentan con bajos recursos de inversión para la infraestructura vial (MINTRANSPORTE, 2011), por esta razón, se crea la necesidad de evaluar nuevos materiales resistentes, de bajo costo de producción y mantenimiento como estabilizantes de vías en afirmado para la red terciaria del país.

Para este trabajo de investigación se planteó la alternativa de usar el residuo de ladrillo proveniente de la Ladrillera Ocaña N.S, como material estabilizante de la vía terciaria no pavimentada que comunica la Ciudad Ocaña con el Corregimiento de Pueblo Nuevo, la cual presenta problemas de plasticidad ante la presencia de lluvias, ocasionando en algunos casos perdidas económicas para los habitantes debido a que el paso por este tramo se vuelve imposible (GarcíaLeón, Flórez Solano, \& Acosta Pérez, 2015).

\section{METODOLOGÍA}

La metodología implementada fue de tipo descriptivo y experimental que tomo como población de estudio, suelos limosos de alta plasticidad de la vía de acceso al Corregimiento de Pueblo Nuevo del Municipio de Ocaña Norte de Santander. La muestra se obtuvo a lo largo de un tramo de $100 \mathrm{~m}$ de subrasante, donde afloran estos materiales, en el sector de estudio. El muestreo se realizó en la vía de acceso al corregimiento de Pueblo Nuevo de la Ciudad de Ocaña (Ver Fig.1), de la cual se tenía previo conocimiento de la existencia de suelos altamente plásticos debido a los resultados obtenidos por Gómez (et al, 2015) con el desarrollo del proyecto de investigación denominado Determinación en la Variación de las Propiedades Mecánicas de los Suelos Arcillosos 
Compresibles en Condición Natural y Estabilizados con Cementantes, el cual reporta que la zona de estudio está compuesta por suelos MH, Limos de alta plasticidad.

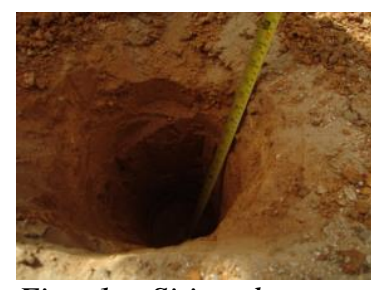

Fig 1. Sitio de toma de muestras. Fuente: Elaboración propia

\subsection{Caracterización Física y Mecánica del suelo en estudio en condiciones naturales}

El programa de ensayos de laboratorio consistió en la realización de pruebas para la clasificación y determinación de propiedades físicas y mecánicas del suelo en condición natural y luego en la mezcla suelo-residuo de ladrillo, los ensayos realizados para la caracterización física fueron Limites de Atterberg, Análisis Granulométrico, gravedad específica y peso volumétrico seco suelto. La Fig. 2 muestra el desarrollo de estos ensayos.

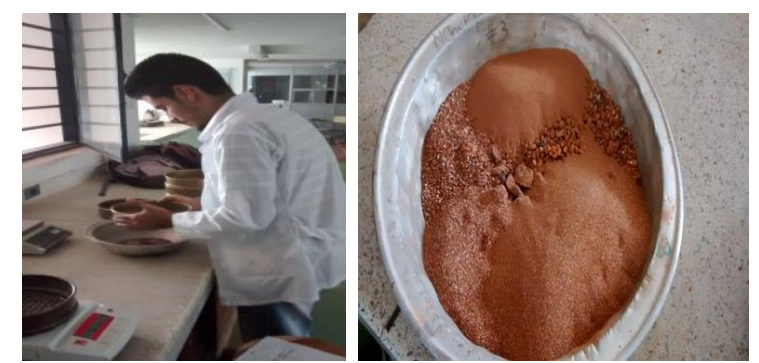

Fig 2. Ejecución de Ensayos de Caracterización Física. uente: Elaboración propia.

Uno de los aspectos fundamentales en el estudio de la subrasante como geomaterial de construcción, es la resistencia mecánica, la cual está gobernada por la sensibilidad del suelo a las variaciones de humedad. Para definir el comportamiento mecánico deben realizarse ensayos sobre muestras representativas, en las cuales se reproducen las condiciones de humedad y densidad que se espera prevalezcan en servicio.

El ensayo más utilizado es el CBR, el cual es una medida de la resistencia del suelo al esfuerzo cortante bajo condiciones de humedad y densidad controladas. (Sánchez, 2014). Otro ensayo de gran importancia es el Proctor, el cual permite conocer el contenido óptimo de humedad para que el suelo alcance la máxima compactación y permitir establecer los parámetros de referencia en el momento de preparar el terreno para el soporte de la cimentación del pavimento. (Valenciano, 2014), en la Fig. 3 se muestra la ejecución de estos ensayos.
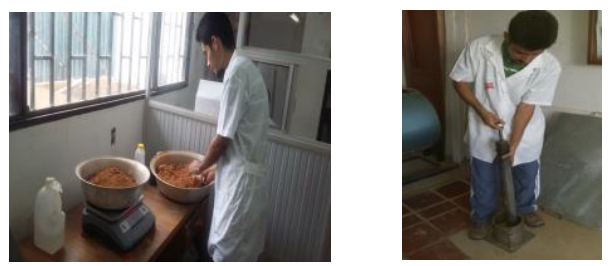

Fig 3. Ejecución de ensayos de caracterización mecánica. Fuente: Elaboración propia.

\subsection{Caracterización física y mecánica de la mezcla suelo- residuo de ladrillo}

Para determinar la dosificación del residuo de ladrillo al suelo, se recurrió a la experiencia del Msc. Álvaro Orlando Pedroza Rojas, el cual ha desarrollado varias propuestas sobre mejoramiento del suelo, entre las cuales se menciona el proyecto denominado Estudio del comportamiento de tres aditivos para estabilizar arcillas expansivas. Cal, cenizas de carbón, colas de rocas fosfóricas. Las dosificaciones utilizadas fueron de $5 \%, 10 \%$ y $15 \%$ en peso.
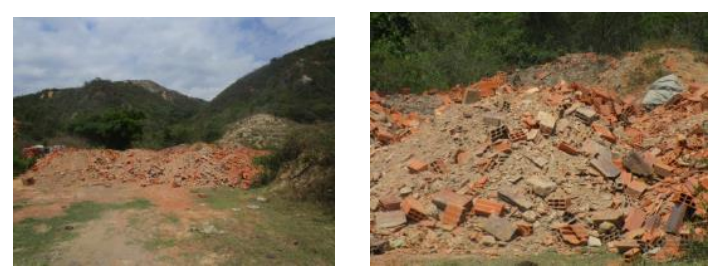

Figura. 4 Forma actual de disposición final del residuo de ladrillo.Fuente: Elaboración propia

Para contribuir a la disposición final de los residuos de la fabricación de ladrillo cerámico por parte de la Ladrillera Ocaña (Ver Fig.4), se implementó el uso de este residuo, como material alternativo para controlar el problema de las subrasantes de alta plasticidad. Según (Payares, 2014) indica que el tipo de arcilla usada para la fabricación del ladrillo en esta Ladrillera son de tres tipos: Una arcilla verde que se clasifica en dos grupos "Verde 1: muestra con menores porcentajes de arenas retenidas sobre las mallas 120,230 y 325 y mayor porcentaje de material pasante sobre la malla 325 y un segundo grupo caracterizado por una arcilla Verde 1A, Verde 1B y Verde 1C: Materias primas que se pueden agrupar por su granulometrías similares" (Caballero A. et al., 2013).

El proceso para disgregar el material, consistió en introducirlo a la máquina de desgastes junto con 6 
esferas a 100 revoluciones, hasta lograr que el material quedara con un tamaño máximo de $12,5 \mathrm{~mm}$, proceso que se puede observar en la Fig.

5 .
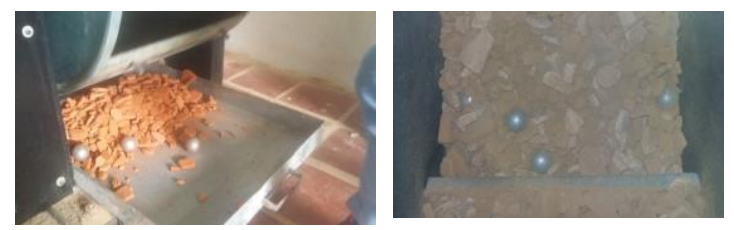

Figura 5. Muestra de estudio en la máquina de los Ángeles. Fuente: Elaboración Propia

\section{* Límites de Atterberg}

Para obtener los resultados de Limite Liquido y Limite Plástico se seleccionó una muestra de $200 \mathrm{gr}$ de suelo natural y posteriormente se le adiciono una porción del residuo de ladrillo dependiendo del porcentaje de dosificación, para luego realizar los ensayos. En la tabla 1 se muestra la cantidad de residuo de ladrillo a usar en este laboratorio.

Tabla 1. Cantidad de muestras a usar. Fuente: Elaboración propia

\begin{tabular}{cccc}
\hline $\begin{array}{c}\text { Peso de la } \\
\text { muestra } \\
\text { condición } \\
\text { natural } \\
\text { (gr) }\end{array}$ & $\begin{array}{c}\text { \% Residuo } \\
\text { de ladrillo }\end{array}$ & $\begin{array}{c}\text { Cantidad } \\
\text { de residuo } \\
\text { de ladrillo } \\
\text { (gr) }\end{array}$ & $\begin{array}{c}\text { Cantidad } \\
\text { total de la } \\
\text { muestra } \\
\text { (gr) }\end{array}$ \\
\hline 200 & 5 & 10 & 210 \\
& 10 & 20 & 220 \\
\hline
\end{tabular}

\section{- Ensayo proctor modificado}

La muestra en estado natural a usar fue obtenida mediante cuarteo, de la cual se tomó 6000gr para luego ser mezclada con la cantidad de residuo de ladrillo descrito en la tabla 2. Esta mezcla fue tamizada por el tamiz No.4 y humedecida.

\section{* Ensayo de CBR}
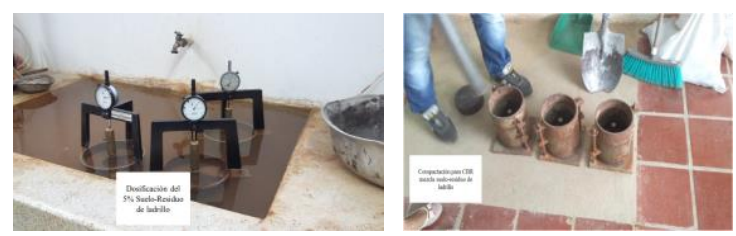

Fig 6. Preparación de ensayo y lectura de deformimetro. Fuente: Elaboración propia.
Tabla 2. Cantidad de muestras a usar Fuente: Elaboración propia

\begin{tabular}{cccc}
\hline $\begin{array}{c}\text { Peso de la } \\
\text { muestra } \\
\text { condición } \\
\text { natural (gr) }\end{array}$ & $\begin{array}{c}\% \\
\text { Residuo } \\
\text { de ladrillo }\end{array}$ & $\begin{array}{c}\text { Cantidad } \\
\text { de residuo } \\
\text { de ladrillo } \\
\text { (gr) }\end{array}$ & $\begin{array}{c}\text { Cantidad } \\
\text { total de la } \\
\text { muestra } \\
\text { (gr) }\end{array}$ \\
\hline 6000 & 5 & 300 & 6300 \\
& 10 & 600 & 6600 \\
& 15 & 900 & 6900
\end{tabular}

La cantidad de suelo y residuo de ladrillo usado para este ensayo se indica en la Tabla 3. Se realizaron tres especímenes para cada una de las dosificaciones, compactados en 5 capas, con golpes 55,26 y 12 golpes, seguidamente fueron sumergidos por cuatro días en los cuales se tomó lecturas del deformímetro y así calcular el porcentaje de expansión y ensayarlos en la maquina multiusos.

Tabla 3. Cantidad de muestra a usar en el ensayo de CBR

\begin{tabular}{cccc}
\hline $\begin{array}{c}\text { Peso de la } \\
\text { muestra } \\
\text { condición } \\
\text { natural } \\
\text { (gr) }\end{array}$ & $\begin{array}{c}\% \\
\text { Residuo } \\
\text { de ladrillo }\end{array}$ & $\begin{array}{c}\text { Cantidad } \\
\text { de residuo } \\
\text { de ladrillo } \\
\text { (gr) }\end{array}$ & $\begin{array}{c}\text { Cantidad } \\
\text { total de la } \\
\text { muestra } \\
\text { (gr) }\end{array}$ \\
\hline 6000 & 5 & 300 & 6300 \\
& 10 & 600 & 6600 \\
\hline & 15 & 900 & 6900 \\
\hline Fuente: Elaboración propia &
\end{tabular}

\section{RESULTADOS}

\subsection{Comportamiento del suelo objeto de estudio en condición natural}

El suelo en estudio caracterizado por un color rojizo con trazas amarillas, es clasificado como un suelo limoso de alta plasticidad (MH) con una gravedad específica de 2.646, en la tabla 4 se encuentra el resumen de los datos obtenidos que fueron base para la clasificación del suelo como MH en el sistema USC y A-7-5 en el sistema AASTHO. 
Tabla 4. Resultados de clasificación del suelo

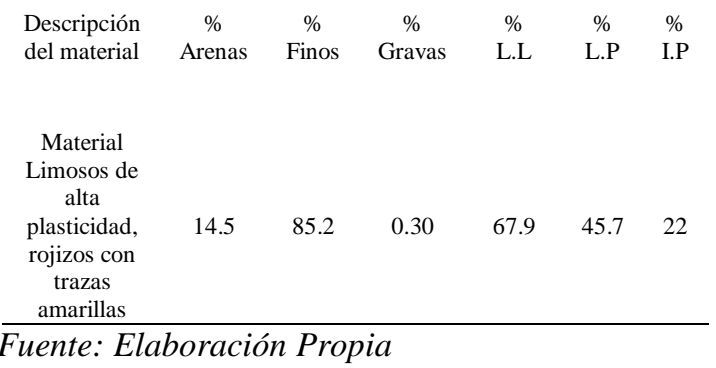

\section{* Ensayo de proctor modificado y CBR}

La tabla 5, muestra los resultados obtenidos en el suelo en estado natural.

Tabla 5. Resumen de propiedades físicas y de capacidad de soporte del suelo

\begin{tabular}{ccccc}
\hline CHO & $\begin{array}{c}\text { Gravedad } \\
\text { Especifica } \\
\text { (Gs) }\end{array}$ & $\begin{array}{c}\text { Densidad } \\
\text { Máxima } \\
(\text { gr/cm3) }\end{array}$ & $\begin{array}{c}\text { \% } \\
\text { CBR } \\
\mathbf{0 . 1}\end{array}$ & $\begin{array}{c}\text { CBR } \\
\text { CBR }\end{array}$ \\
\hline \multirow{2}{*}{32.55} & 2.646 & 1.407 & 6.7 & 5.1 \\
\hline
\end{tabular}

Fuente: Elaboración propia

\subsection{Comportamiento del suelo objeto de estudio en condición suelo-residuo de ladrillo}

Los parámetros evaluados son las propiedades índices y mecánicas del suelo con la adición de residuo de ladrillo y posteriormente compararlo con las calculadas en condición natural.

a. Límites de Atterberg de la muestra suelo residuo de ladrillo

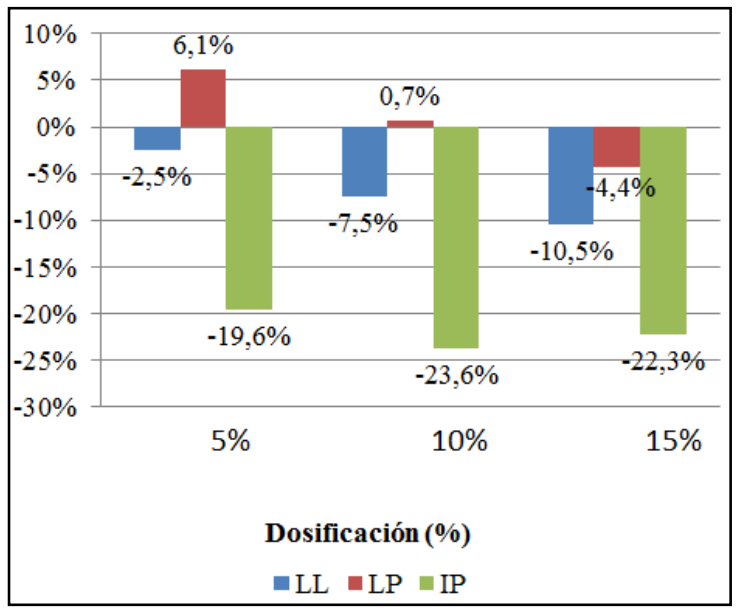

Fig 7. Variación porcentual límites de consistencia residuo de ladrillo. Fuente: Elaboración propia
En la Fig. 7 Se muestran la variación porcentual obtenida en los ensayos de límites de consistencia en la mezcla suelo-residuo de ladrillo, en la cual se puede observar que el limite líquido y plástico disminuyeron hasta un $10.46 \%$ y un $4.38 \%$ respectivamente, referente a la condición natural, lo cual muestra que el residuo de ladrillo tiene un efecto en la reducción de la plasticidad del suelo.

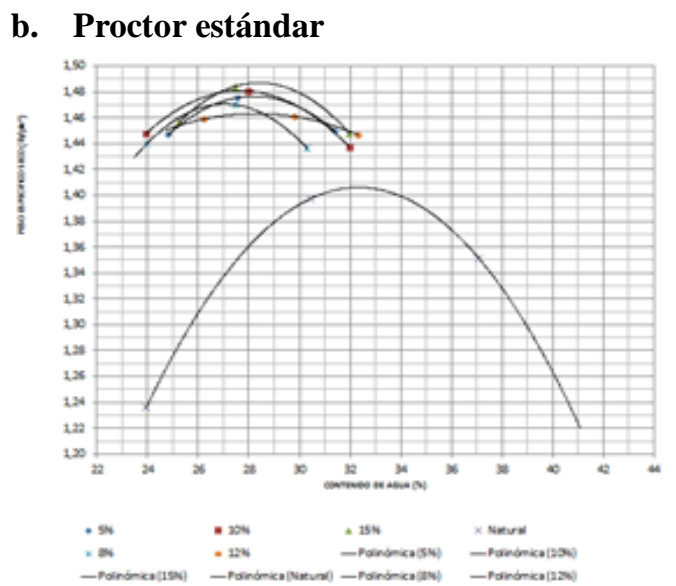

Fig 8. Variación de densidad seca y humedad optima con las diferentes dosificaciones. Fuente: Elaboración propia.

En la Fig. $8 \mathrm{Se}$ aprecia el comportamiento de las curvas de humedad vs densidad seca de las mezclas respecto a la curca para la muestra sin adición de cementante. En la Fig. 9 se muestra la variación porcentual del peso específico seco y la humedad óptima del suelo en estado natural y en las diferentes dosificaciones de la mezcla sueloresiduo de ladrillo, donde para la dosificación del $15 \%$ la mezcla tiene un aumento máximo del $5.83 \%$ en el peso específico seco. En relación a la humedad óptima se observa que la máxima disminución se obtuvo con una dosificación del $10 \%$ igual a $14,29 \%$.

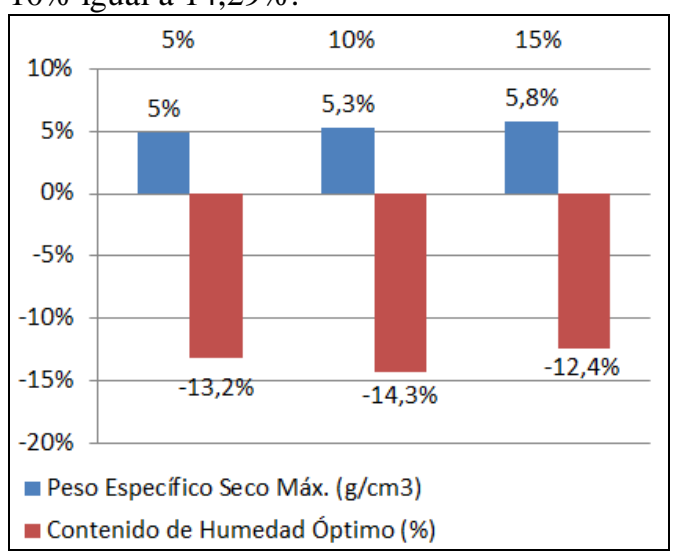

Fig 9. Variación porcentual de la mezcla sueloresiduo de ladrillo en el ensayo de proctor estándar. Fuente: Elaboración propia 


\section{c. Ensayo de CBR de Laboratorio}

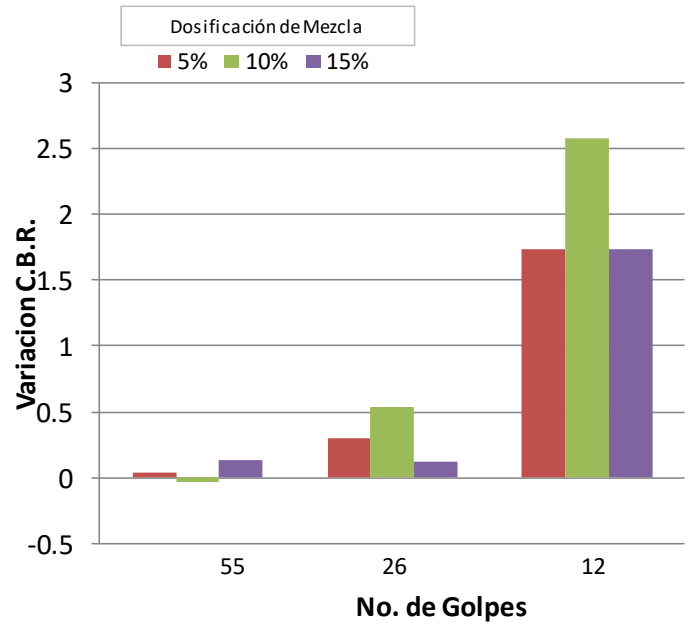

Fig 10. Variación porcentual del Índice CBR Fuente: Elaboración propia

En la Fig. 10 se encuentra la variación porcentual entre los valores de CBR del suelo en estado natural con los valores de las mezcla de suelo mezclado con residuo de ladrillo en sus diferentes dosificaciones; donde se resalta un aumento del valor de CBR en un 3\% del CBR de la dosificación de la mezcla del $10 \%$.

\section{CONCLUSIONES}

Los resultados obtenidos en esta investigación son experimentales debido a que fueron ensayados en condiciones controladas, para verificar su efectividad deberán ser comprobados en obras y/o tramos viales

La mezcla suelo-residuo de ladrillo representa una buena opción para el mejoramiento de suelos altamente plásticos, pues se encontró que se puede bajar el índice de plasticidad hasta en un 22,27\% para una dosificación del 10\% de esta mezcla. Además en otros parámetros como la densidad máxima seca un aumento de $5,83 \%$ en una dosificación del $15 \%$ y la humedad optima una disminución del 14,29\% para dosificación del $10 \%$. La respuesta de la mezcla de suelo-residuo de ladrillo en relación al índice CBR, no muestra una mejora considerable siendo esta de máximo el 3\% con respecto a la condición natural.

El uso de materiales alternativos como el residuo de la fabricación del ladrillo permite no solo obtener una mejora en el comportamiento físico del suelo sino también contribuir a la protección del medio ambiente al poder disponer de este material evitando una posible contaminación por arrastres de sedimentos de la principal fuente hídrica de la Ciudad de Ocaña, Norte de Santander.

\section{REFERENCIAS}

Caballero Amaury, Velasco Gabriel, Pardo García A. (2013). DIFFERENTIATIONS OF OBJECTS IN DIFFUSE DATABASES. Revista colombiana de tecnologías de Avanzada. 2 (22). Pág. 131 - 137.

Carvajal P. Gloria I, Arias J. Yhan P (2015), Valoración de las cenizas de carbón para la estabilización de suelos mediante activación alcalina y su uso en vías no pavimentadas, tesis de pregrado Universidad de Medellín.

Das, B. (1999). Fundamentos de Ingeniería Geotécnica. Ed. Thomson Learning. México D.F

MINTRANSPORTE. (2011). Diagnóstico del Transporte. Ministerio de Transporte.

Payares P. Carlos A. (2014). Estudio de la relación entre las propiedades físicas finales de los bloques cerámicos para mampostería y los perfiles de temperatura presentados en el horno durante su cocción en la ladrillera Ocaña, tesis de pregrado, Universidad Francisco de Paula Santander Ocaña, Facultad de Ingenierías.

Pedroza R. Álvaro O. (1986). Estudio del comportamiento de tres aditivos para estabilizar arcillas expansivas. Cal, cenizas de carbón, colas de rocas fosfóricas.

García-León, R. A., Flórez Solano, E., \& Acosta Pérez, M. A. (2015). Análisis estructural de una máquina prensadora para producción de ladrillo macizo para las pequeñas industrias artesanales de materiales cerámicos en Ocaña Norte de Santander y en la región. Revista Colombiana de Tecnologías de Avanzada, 1(1692-7257), 7.

SITIOS WEB

Fededesarrollo (2013), Indicadores del sector transporte en Colombia, 2013, consultado el 07 de Marzo de 2016 en http://www.fedesarrollo.org.co/wpcontent/uploads/2011/08/Indicadores-delsector-transporte-en-Colombia-InformeConsolidado.pdf

Tamayo T. Jorge (1985), Mejoramiento del comportamiento de suelos y materiales con la incorporación de aditivos no convencionales, 24-32, Ingeniería e Investigación, Vol.3 No.2, Universidad Nacional. Recuperado de file:///F:/Dialnet-

MejoramientoDelComportamientoDeSuelos YMaterialesCo-4902444\%20(1).pdf 